\title{
BMJ Open KMBARC registry: protocol for a multicentre observational cohort study on non-cystic fibrosis bronchiectasis in Korea
}

\begin{abstract}
Hyun Lee, ${ }^{1}$ Hayoung Choi (D) , ${ }^{2}$ Yun Su Sim, ${ }^{2}$ Shinhee Park, ${ }^{3}$ Woo Jin Kim, ${ }^{4}$ Kwang Ha Yoo, ${ }^{5}$ Seung Jun Lee, ${ }^{6}$ Tae-Hyung Kim, ${ }^{7}$ Bumhee Yang, ${ }^{8}$ Ina Jeong, ${ }^{9}$ Soo-Jung Um, ${ }^{10}$ Deog Kyeom Kim, ${ }^{11}$ Ji-Hyun Lee, ${ }^{12}$ Byoung Soo Kwon, ${ }^{13}$ Young-Jae Cho, ${ }^{13}$ Hye Yun Park, ${ }^{14}$ Chang-Hoon Lee, ${ }^{15}$ Chin Kook Rhee, ${ }^{16}$ Sang Haak Lee, ${ }^{17}$ Ju Ock Na, ${ }^{18}$ An-Soo Jang, ${ }^{19} \mathrm{Ji}$ Ye Jung, ${ }^{20}$ Seung Won $\mathrm{Ra},{ }^{21}$ $\mathrm{Ji}$-Ho Lee, ${ }^{22}$ Sang-Ha Kim, ${ }^{22}$ Changhwan Kim, ${ }^{23}$ Youlim Kim, ${ }^{24}$ Chang Youl Lee, ${ }^{24}$ Hyun Kuk Kim, ${ }^{25}$ Jae Seung Lee, ${ }^{26}$ Sei Won Lee, ${ }^{26}$ Yeon-Mok Oh ${ }^{10},{ }^{26}$ on behalf of the KMBARC
\end{abstract}

To cite: Lee H, Choi H, Sim YS, et al. KMBARC registry: protocol for a multicentre observational cohort study on non-cystic fibrosis bronchiectasis in Korea. BMJ Open 2020;10:e034090. doi:10.1136/ bmjopen-2019-034090

\section{- Prepublication history and} additional material for this paper are available online. To view these files, please visit the journal online (http://dx.doi org/10.1136/bmjopen-2019034090).

$\mathrm{HL}$ and $\mathrm{HC}$ contributed equally.

Received 05 September 2019 Revised 10 December 2019 Accepted 17 December 2019

Check for updates

(C) Author(s) (or their employer(s)) 2020. Re-use permitted under CC BY-NC. No commercial re-use. See rights and permissions. Published by BMJ.

For numbered affiliations see end of article.

Correspondence to

Dr Yeon-Mok Oh;

yeonmok.oh@gmail.com

\section{ABSTRACT}

Introduction Despite the significant disease burden of bronchiectasis in Korea, no large-scale, representative prospective cohort studies have been conducted to evaluate the clinical characteristics of Korean patients with bronchiectasis, indicating an urgent need for cohort studies on bronchiectasis.

Methods and analysis The Korean Multicenter Bronchiectasis Audit and Research Collaboration (KMBARC) is a prospective, non-interventional observational cohort study on bronchiectasis in Korea. The inclusion criteria of this registry are as follows: (1) adult patients (aged $\geq 18$ years) with or without respiratory symptoms (cough, chronic sputum and/or recurrent respiratory infection) and chest computed tomography revealing bronchiectasis affecting one or more lobes and (2) stable status at the time of registration: patients with bronchiectasis who were admitted for a respiratory aetiology can be enrolled at least 4 weeks after hospital discharge. The exclusion criteria are as follows: (1) bronchiectasis due to cystic fibrosis; (2) traction bronchiectasis associated with interstitial lung disease; (3) patients actively being treated for pneumonia, pulmonary tuberculosis or nontuberculous mycobacterial infection; (4) patients who are unable or unwilling to provide informed consent; and (5) pregnant patients. Although the KMBARC questionnaires for baseline and annual follow-up data are similar to the European Multicentre Bronchiectasis Audit and Research Collaboration questionnaires, KMBARC has distinctive features such as use of Bronchiectasis Health Questionnaires, measurement with fatigue and depression scales, blood tests, use of consensus definition of exacerbations and information on emergency room or hospitalisation.

We aim to recruit at least 1200 patients over the study period from more than 26 hospitals in South Korea. Patients will undergo a detailed baseline and yearly assessment for up to 5 years. The study objectives of the KMBARC registry are as follows: (1) uncovering the
Strengths and limitations of this study

This is the first prospective cohort study on patients with bronchiectasis in Korea.

- We will recruit and follow-up patients with bronchiectasis annually using a standardised protocol to improve the quality of data collection.

- Sharing similar case-reporting forms with European Multicentre Bronchiectasis Audit and Research Collaboration (EMBARC) will allow collaboration studies with EMBARC.

- Distinctive features of the Korean Multicenter Bronchiectasis Audit and Research Collaboration registry (KMBARC) will provide several novel findings of bronchiectasis, which might be difficult to be elucidated using other registries.

- This study is limited by a lack of collecting patient samples.

natural course of bronchiectasis; (2) aiding in establishing evidence-based bronchiectasis guidelines in Korea; and (3) encouraging and facilitating studies on bronchiectasis in Korea.

Ethics and dissemination This study received necessary approval from the Institutional Review Boards of all participating institutions. The Asan Medical Center Institutional Review Board gave overall approval for the study. Results will be disseminated via peer-reviewed publications and conference presentations. Trial registration number KCT0003088.

\section{INTRODUCTION}

Bronchiectasis is a chronic respiratory disease characterised by irreversible bronchial dilatation. ${ }^{1}$ Patients with bronchiectasis often develop chronic respiratory 
symptoms, including cough, purulent sputum, haemoptysis, dyspnoea and recurrent infection. ${ }^{1}$ This disease is associated with substantial health-related burden, such as a substantially low quality of life, ${ }^{2}$ increased healthcare costs, ${ }^{3}$ increased frequency of hospitalisation and mortality; however, bronchiectasis has been neglected by pulmonologists and regarded as an 'orphan disease'.

A surge in interest in bronchiectasis has been noted with the initiation of international observational studies such as the European Multicentre Bronchiectasis Audit and Research Collaboration (EMBARC) ${ }^{4}$ and the US Bronchiectasis Registry research. ${ }^{5}$ While these cohort studies have unravelled several important aspects of bronchiectasis, such as epidemiology, factors leading to disease exacerbations, quality of life and mortality, ${ }^{5}$ they lack inclusivity. Specifically, only few Asian patients have been included, and considering the prevalence and disease burden of bronchiectasis in these patients, which are more substantial than those in the Western population, ${ }^{6}$ there is an urgent need to evaluate the clinical characteristics, aetiologies, comorbidities and prognosis of this disease among Asian populations, including the Korean population. We have established the Korean Multicentre Bronchiectasis Audit and Research Collaboration (KMBARC) to: (1) evaluate the clinical characteristics and phenotypes of bronchiectasis in Korea; (2) promote clinical studies on bronchiectasis in Korea; and (3) improve patient care and education among Korean patients with bronchiectasis. The protocol for the KMBARC registry is described herein.

\section{METHODS AND ANALYSIS \\ Study objectives}

The objective of this study is to develop the KMBARC database with baseline and annual follow-up data for at least 5 years. The specific study objectives will be as follows:

1. Description of the clinical characteristics, including patient demographics, phenotype, aetiology, progression, treatment and prognosis, of Korean patients with bronchiectasis.

2. Evaluation of disease burden, including use of medication and medical resources, acute exacerbation, hospitalisation and mortality, in Korean patients with bronchiectasis.

3. Evaluation of the rare aetiology of bronchiectasis (eg, allergic bronchopulmonary aspergillosis, rheumatoid arthritis and tuberculosis).

4. Elucidation of risk factors associated with acute exacerbation and prognosis.

\section{Registry design}

The KMBARC registry is a multicentre, prospective, non-interventional, observational cohort study enrolling consecutive adult patients with non-cystic fibrosis bronchiectasis in Korea. The framework for the development of the KMBARC registry protocol and case-reporting forms is obtained from the EMBARC registry protocol with permission of the EMBARC leadership. ${ }^{4}$ Therefore, the information in the KMBARC registry is very similar to that in the EMBARC registry with some modificationsinformation specific to the Korean bronchiectasis registry was included. Given the nature of observational studies, management of bronchiectasis has been performed at the discretion of attending physicians in each local hospital participating in the registry. Therefore, participating researchers were recommended to follow the most recently released European Respiratory Society guidelines for the management of adult bronchiectasis ${ }^{1}$ in their daily practice to synchronise and standardise the process prior to initiation of the study.

\section{Study participants}

The inclusion criteria of this registry are as follows: (1) adult patients (aged $\geq 18$ years) with or without respiratory symptoms (cough, chronic sputum and/or recurrent respiratory infection) and having chest computed tomography (CT) results demonstrating bronchiectasis affecting one or more lobes; (2) stable status at the time of registration; patients with bronchiectasis who were admitted due to respiratory aetiology can be enrolled at least 4 weeks after hospital discharge.

The exclusion criteria are as follows: (1) bronchiectasis due to cystic fibrosis; (2) traction bronchiectasis associated with interstitial lung disease; (3) patients actively being treated for pneumonia, pulmonary tuberculosis or non-tuberculous mycobacterial infection; (4) patients who are unable or unwilling to provide informed consent; and (5) pregnant patients.

\section{Patient recruitment and follow-up}

Patients will be identified and recruited at outpatient clinics in 26 specialist hospitals in Korea, which include the Asan Medical Center, Hanyang University Seoul Hospital, Hallym University Kangnam Sacred Heart Hospital, Gangneung Asan Hospital, Kangwon National University Hospital, Konkuk University Hospital, Gyeongsang National University Hospital, Hanyang University Guri Hospital, National Cancer Center, National Medical Center, Dong-a University Hospital, Seoul Metropolitan Government-Seoul National University Boramae Medical Center, CHA Bundang Medical Center, Seoul National University Bundang Hospital, Samsung Medical Center, Seoul National University Hospital, Catholic University Seoul St. Mary's Hospital, Catholic University St. Paul's Hospital, Soonchunhyang University Cheonan Hospital, Soonchunhyang University Bucheon Hospital, Yonsei University Severance Hospital, Ulsan University Hospital, Wonju Severance Christian Hospital, Jeju National University Hospital, Hallym University Chuncheon Sacred Heart Hospital and Inje University Haeundae Paik Hospital.

If there are other hospitals that want to participate in the KMBARC registry in the future, the number of hospitals can be increased. Patients will be followed up at least once a year. During the year, the frequency of outpatient 
clinic visits will be determined at the discretion of the attending physicians.

\section{Data collection}

Data will be collected from three distinct fields as follows:

(1) baseline data, (2) annual follow-up data and (3) data during hospitalisation. The KMBARC questionnaires for baseline and annual follow-up data are similar to those of EMBARC. $^{4}$

Distinctive features of the KMBARC registry are as follows:

1. Patients without daily respiratory symptoms will be included.

2. Bronchiectasis Health Questionnaires (BHQs) will be used to evaluate quality of life instead of using the 'Quality of Life-Bronchiectasis' questionnaire.

3. Baseline information of the Patient Health Questionnaire 9 (PHQ-9) ${ }^{7}$ and the Fatigue Severity Score $(\mathrm{FSS})^{8}$ will be assessed to evaluate the status of depression and fatigue in patients with bronchiectasis, respectively.

4. Prebronchodilator and/or postbronchodilator spirometry will be measured to evaluate lung function.

5. Use of macrolides.

6. Patients' residential addresses will be collected to compare the clinical characteristics between patients with bronchiectasis who live in urban and rural areas.

7. Complete blood count, including white cell count (neutrophil, eosinophil, monocyte and so on), haemoglobin and platelet, erythrocyte sedimentation rate, C-reactive protein, total bilirubin, uric acid, blood urea nitrogen and creatinine, will be determined from collected blood samples.

8. A medical history of measles will be noted.

9. A consensus definition of acute exacerbations of bronchiectasis $^{9}$ will be used. Because our definition includes exacerbations requiring antibiotics and treatment modification other than antibiotics, we will collect information on antibiotic use during acute exacerbations.

10. Information on date of emergency room (ER) visit/ hospitalisation, admission to intensive care unit, use of mechanical ventilator, aetiology of ER visit/hospitalisation (haemoptysis, pneumonia or other causes), development of arrhythmia (except for atrial tachycardia), bronchial embolisation, level of brain natriuretic peptide, respiratory virus (detected by a nucleic acid amplification test) and influenza A/B antigen (box 1) will be evaluated.

\section{Definition of exacerbation}

Acute exacerbations are defined as deterioration in $\geq 3$ of the following symptoms for at least 48 hours: (1) cough; (2) sputum volume increase and/or consistent change; (3) sputum purulence; (4) dyspnoea and/or exercise intolerance; (5) fatigue and/or malaise; and (6) haemoptysis. ${ }^{9}$ Severe exacerbations are defined as exacerbations requiring an ER visit or hospitalisation. ${ }^{10}$ To
Box 1 Clinical data collected at baseline, follow-up and exacerbations

Baseline

- Enrolment date.

$>$ Age.

Body mass index.

> Comorbidities.

- Pulmonary function tests.

- Modified Medical Research Council dyspnoea scale.

- Smoking history.

- Exacerbation history 1 year before study enrolment.

- Medical history and laboratory examination to determine the aetiology of bronchiectasis.

- Aetiology of bronchiectasis determined by attending physicians.

- Microbiological examination results.

CT findings in bronchiectasis.

- Respiratory medications.

- Respiratory physiotherapy.

- Bronchiectasis Health Questionnaire (BHQ).

- Patient Health Questionnaire 9 (PHQ-9).

Fatigue Severity Score (FSS).

\section{Follow-up}

- Follow-up date.

- Survival.

- Body mass index.

- Comorbidities.

- Smoking history.

- Exacerbations.

- Updated medical history and laboratory examination to determine the aetiology of bronchiectasis.

- Microbiological examination results.

- CT findings of patients with bronchiectasis.

- Respiratory medications.

- Respiratory physiotherapy.

- BHQ.

\section{Exacerbations}

- Admission or emergency room visit date.

- Mechanical ventilator use.

- Intensive care unit admission.

- Arrhythmia.

- Laboratory examination results.

- Reasons for admission.

- Respiratory virus (detected by a nucleic acid amplification test) and influenza A/B antigen.

- Influenza antigen test.

identify acute exacerbations that require antibiotics, we will collect information on the use of antibiotics in the events of acute exacerbations.

\section{Sputum assessment}

Sputum colour and volume $(\mathrm{mL} /$ day) in patients with stable bronchiectasis will be assessed. Sputum colour is evaluated using a validated, photographic sputum colour chart-the colour is rated as 1 (mucoid), 2 (mucopurulent), 3 (purulent) or 4 (severely purulent). ${ }^{11}$ Patient sputum volume will be estimated based on reference 
volumes of $50 \mathrm{~mL}$ with a small glass and $200 \mathrm{~mL}$ with a cup.

\section{Aetiology of bronchiectasis}

The attending physicians will determine the aetiology of bronchiectasis based on test results and questionnaires. The aetiologies include idiopathic, postinfectious, posttuberculosis, allergic bronchopulmonary aspergillosis, rheumatoid arthritis, connective tissue disease, inflammatory bowel disease, aspiration, gastro-oesophageal reflux disease, non-tuberculous mycobacterium pulmonary disease, chronic obstructive pulmonary disease (COPD), asthma, primary ciliary dyskinesia, Kartagener's syndrome, Young's syndrome, $\alpha-1$ antitrypsin deficiency, common variable immunodeficiency, X-linked agammaglobulinaemia, immunoglobulin A deficiency, immunoglobulin G subclass deficiency, specific antibody deficiency, human immunodeficiency virus infection, Williams-Campbell syndrome, Marfan syndrome, Mounier-Kuhn syndrome, yellow nail syndrome, human T-lymphotropic virus type 1 infection, infant chronic lung disease, mechanical ventilation in newborn infant and pink disease. Physicians can choose multiple aetiologies and determine a specific aetiology other than the above suggested aetiologies (eg, diffuse panbronchiolitis).

\section{Lung function}

Prebronchodilator and/or postbronchodilator spirometry will be performed according to American Thoracic Society/European criteria. ${ }^{12}$ Absolute values of forced expiratory volume in $1 \mathrm{~s}\left(\mathrm{FEV}_{1}\right)$ and forced vital capacity (FVC) will be recorded, and the percentage of predicted values for $\mathrm{FEV}_{1}$ and FVC will be calculated using an automatic calculator using a reference equation obtained in a representative Korean sample. ${ }^{13}$ In addition to prebronchodilator and/or postbronchodilator spirometry, lung function tests results, including those of total lung capacity, diffusing capacity, residual volume or inspiratory capacity, are collected if available.

\section{Severity of bronchiectasis}

The severity of bronchiectasis is evaluated using the bronchiectasis severity index calculated by an automatic calculator incorporated in the database ${ }^{14}$ and based on essential variables.

\section{Comorbidities}

Regarding pulmonary comorbidities, the presence of physician-diagnosed asthma, COPD, nasal polyp and chronic sinusitis will be assessed. Extrapulmonary comorbidities such as cardiovascular diseases, metabolic diseases, chronic liver diseases, osteoporosis, depressive disorder, anxiety disorder, chronic kidney diseases and malignancy will also be assessed.

\section{Microbiology}

The microbiological profiles of the spontaneous sputum specimen and lower respiratory tract specimen (induced sputum, bronchoalveolar lavage or nasopharyngeal swab) will be determined; sputum cultures from clinically stable patients and those with acute exacerbation of the disease will be analysed according to standard methods. ${ }^{15}$ Pseudomonas aeruginos $a$ is significantly associated with morbidity and mortality in bronchiectasis ${ }^{16}{ }^{17}$; therefore, specific data on $P$. aeruginosa such as the timing of growth; strain type (mucoid, nonmucoid or unknown); and the use of oral, intravenous or inhaled antibiotics for eradication will be obtained. In addition, the growth of Mycobacterium tuberculosis and non-tuberculous mycobacterium will be determined.

\section{Radiology}

The severity of dilatation and the number of involved lobes will be measured to evaluate the severity of bronchiectasis using the modified Reiff score, which has been shown to be correlated with bronchiectasis-related hospital admissions and mortality. ${ }^{18}{ }^{19}$ Attending physicians will determine radiological severity (no bronchiectasis, tubular, varicose, cystic or unknown) in each lobe (right upper, right middle, right lower, upper division of left upper, lingular division of left upper and left lower). Unknown severity indicates that the attending physicians confirmed the presence of bronchiectasis on the basis of a radiologist's formal reading, but the attending physician cannot determine the severity because of unavailability of chest CT recordings.

\section{Treatment}

We will assess whether patients with bronchiectasis receive regular respiratory treatment comprising the following:

1. Respiratory medications: long-acting muscarinic antagonist (LAMA), inhaled corticosteroid (ICS), longacting $\beta_{2}$ agonist (LABA), ICS/LABA, LAMA/LABA, intravenous immunoglobulin, itraconazole, leukotriene receptor antagonist, long-term ( $\geq 28$ days) use of oral steroid, monoclonal antibody, mucoactive drug or nebulised bronchodilator.

2. Antibiotics: inhaled antibiotics, long-term ( $\geq 28$ days) use of oral antibiotics or systemic antibiotics.

3. Physiotherapy or mucoactive treatment, including nebulised saline, nebulised hypertonic saline, sodium hyaluronate, nebulised mannitol or DNase.

Data on the use of long-term home oxygen therapy, noninvasive ventilation or oral theophylline will be collected.

\section{Quality control and grading system}

Automated logic checks are incorporated to prevent outof-range values being entered or to provide feedback warnings to users when data are out of range. Each case entered into the registry is manually verified by study team members, and data queries are resolved with members at the study site.

To maintain the highest quality of data, we will use a grading system wherein all clinical variables are categorised into essential (10 variables), important (125 variables) and less important (28 variables) categories. Based on the ratio of filled up data to each category, the quality 
of each case data will be graded A, B, C or D. Study team members will be encouraged to enter essential as well as important variables to improve the quality of data according to the grading system. Cases with missing data for essential variables (or unresolved queries) or those classified as $\mathrm{D}$ will be rejected to retain only high-quality data in the registry.

\section{Sample size}

To calculate the sample size, we assumed a proportional hazard model for the development of the first exacerbation with the following assumptions: (1) patients with a major phenotype of bronchiectasis account for $20 \%$ of all study patients; (2) patients with a major phenotype have a relative hazard of exacerbation value of 0.80 ; (3) alpha error is 0.05 and beta error is 0.20 ; (4) average exacerbation rate is 1.5 events per year; and (5) average duration of follow-up is 3 years with censoring rate of 0.30 . Based on these assumptions, the total calculated sample size was 1200 patients, and the sample size for the group of patients with the major phenotype was 240 patients. We set a target of 1200 patients to be enrolled by May 2020 . A short-term target of 400 patients in the first year of the project (up to August 2019) has been set for the analysis of baseline characteristics. To evaluate bronchiectasis in Korean patients, we aim to include patients from 26 Korean hospitals.

\section{Governance, oversight and data sharing}

The registry collects data using Electronic Data Capture solution, a web-based data management system that shares data through a web browser. The study data are held securely on the server of the Internet Data Center, which has a firewall system to ensure physical and technical data security. Anonymised data will be accessible to researchers in Korea, and investigators can access data without restrictions. However, complete data analysis requires submission of a research proposal to the KMBARC Scientific Committee. Access will be granted by the KMBARC scientific committee after approval of the proposed research. Although open data sharing is the basic principle of the KMBARC registry, unreasonable request for the data (eg, commercial purposes) can be rejected by the scientific committee.

\section{Patient involvement}

Patients were not involved in setting the research question, the outcome measures, the design or implementation of the study. Patients will not be involved in the recruitment to and conduct of the study. We plan to translate the results into short, easy-to-read summaries and to disseminate it to the relevant patient community through local media.

\section{ETHICS AND DISSEMINATION}

The study is performed in accordance with the principles of Good Clinical Practice. This study protocol was approved by the Institutional Review Board at each site (refer to online supplementary file 1 for the list). The Asan Medical Center Institutional Review Board gave overall approval for the study. Written informed consent should be obtained from all patients. The KMBARC study group will follow the recommendations regarding authorship provided by the International Committee of Medical Journal Editors. Results will be disseminated via peerreviewed publications and conference presentations.

\section{DISCUSSION}

The KMBARC registry is the first prospective multicentre cohort study to evaluate the clinical characteristics, natural course and prognosis of bronchiectasis in Korea. Through a comprehensive evaluation of patients with bronchiectasis in Korea, we expect the KMBARC registry will generate hypotheses to solve research questions in bronchiectasis and information to enable physicians assess aetiology and manage patients with bronchiectasis in real-world settings. From this perspective, the KMBARC registry will pave the way for improvements in bronchiectasis management and research collaboration.

Despite the lack of epidemiological data in Korea, epidemiological studies have demonstrated that bronchiectasis poses a significant burden on public health, including increased healthcare costs, hospital admission and mortality. ${ }^{20-22}$ Therefore, we conducted a nationwide database study on the prevalence of bronchiectasis in Korea. ${ }^{6}$ Our study demonstrated that the prevalence of non-cystic fibrosis bronchiectasis was 464 cases per 100000 population, which was strikingly higher than that reported in Germany (67 cases per 100000 population) and the USA (138 cases per 100000 population). ${ }^{23}{ }^{24}$ Despite the higher disease burden of bronchiectasis in Korea than that in Western counties, there is a lack of data on baseline characteristics (aetiology, clinical presentation, quality of life, treatment modality and exacerbation rates) of bronchiectasis in Korea. Previous studies have suggested that idiopathic aetiology and respiratory infections, including pulmonary tuberculosis, are the leading causes of bronchiectasis in Asian patients. ${ }^{25-29}$ Other than the causes of bronchiectasis, few studies have investigated the baseline characteristics of Korean patients with bronchiectasis, which will likely be revealed through analysis of the KMBARC registry data. From this perspective, we acknowledge the recently published study by Dhar et al, ${ }^{30}$ which investigates the differences between patients with bronchiectasis in India and those in Europe. Compared with European patients, Indian patients showed a high frequency of severe, cystic bronchiectasis. Tuberculosis and other severe infections were the most frequently reported conditions as underlying causes of bronchiectasis. In line with this, we believe that the KMBARC registry will add more evidence to the Asian phenotypes of bronchiectasis. In addition, a collaboratory study on participants of the KMBARC registry and Indian participants of the EMBARC registry would provide valuable 
information about clinical differences in region and race among Asian patients with bronchiectasis.

One of the most important advantages of the cohort study is that it allows long-term follow-up of patients to monitor disease progression and track treatment response and prognosis (acute exacerbation, hospitalisation and mortality). However, in contrast to other respiratory diseases such as COPD, ${ }^{31-36}$ the number of cohort studies on bronchiectasis with large sample size is limited, especially in Asian countries. Thus, through prospective cohort studies, the KMBARC registry will provide in-depth information on the natural course of specific phenotypes of bronchiectasis. The registry will also provide valuable longitudinal data regarding acute exacerbation and hospitalisation. A potential benefit of the KMBARC registry is that it may encourage and facilitate studies on bronchiectasis in Korea (eg, translational research and randomised controlled trial beyond cohort study). We also expect that the KMBARC registry will be helpful to identify hospitals that are most likely to contribute to clinical trials. ${ }^{37}$

The lack of a well-established bronchiectasis cohort has also led to our overall ignorance of real-world practice in the diagnosis, treatment and prognosis of bronchiectasis in the Korean population. It is expected that the KMBARC registry will help identify common diagnostic approaches and treatment modalities among Korean patients with bronchiectasis, enable the establishment of strategies to diagnose and treat based on evidence-based medicine for Korean patients with bronchiectasis and improve the quality of real-world daily practice.

Because the KMBARC registry is based on the framework of the EMBARC protocol, collaborative studies involving the two may help unravel racial differences in the aetiologies and phenotypes of bronchiectasis as well as bronchiectasis comorbidities. ${ }^{4}$ There is reason to suspect racial and ethnic differences in many aspects of bronchiectasis since we have demonstrated lower prevalence of COPD and cardiovascular diseases in the Korean population than in the Western population. ${ }^{638}$ However, the reasons for these ethnic differences remain unclear due to insufficient information contained in the national health insurance database.

In addition to the shared framework of the EMBARC, the KMBARC has many distinctive features. First, this study enrols patients with bronchiectasis without daily respiratory symptoms, which may have a potential advantage over the EMBARC, including only symptomatic patients with bronchiectasis. Second, additional questionnaires such as PHQ-9 and FSS may unveil in-depth information on depression and fatigue in those with bronchiectasis. Third, the KMBARC has postbronchodilator spirometry results, which may provide useful information on the role of inhalers in those with bronchiectasis. It has been suggested that positive bronchodilator response can predict responsiveness to long-term bronchodilator therapy in patients with bronchiectasis. ${ }^{39}$ Fourth, the KMBARC has laboratory examination results such as neutrophil and eosinophil count, total bilirubin, uric acid, erythrocyte sedimentation rate and C-reactive protein. The analysis of clinical characteristics, exacerbation history and radiological findings along with the blood test results may give us an insight into the phenotypes of bronchiectasis.

In conclusion, the KMBARC aims to recruit 1200 patients from more than 26 hospitals in South Korea by May 2020. Our cohort will provide information on phenotype, natural course, aetiology, exacerbation and prognosis of bronchiectasis. Furthermore, the KMBARC will facilitate research collaboration and meaningful studies of bronchiectasis and generate enough data to support the establishment of evidence-based clinical practice guidelines for bronchiectasis in Korea.

\section{Author affiliations}

${ }^{1}$ Division of Pulmonary Medicine and Allergy, Department of Internal Medicine, Hanyang University Hospital, Seoul, Korea (the Republic of)

${ }^{2}$ Division of Pulmonary, Allergy, and Critical Care Medicine, Department of Internal Medicine, Hallym University Kangnam Sacred Heart Hospital, Seoul, Korea (the Republic of)

${ }^{3}$ Department of Pulmonary and Critical Care Medicine, Gangneung Asan Hospital, Gangneung, Korea (the Republic of)

${ }^{4}$ Department of Internal Medicine and Environmental Health Center, Kangwon National University, Chuncheon, Korea (the Republic of)

${ }^{5}$ Department of Internal Medicine, Konkuk University School of Medicine, Seoul, Korea (the Republic of)

${ }^{6}$ Department of Internal Medicine, Gyeongsang National University Hospital, Gyeonsang National University School of Medicine, Jinju, Korea (the Republic of) ${ }^{7}$ Division of Pulmonary Medicine and Allergy, Department of Internal Medicine, Hanyang University Guri Hospital, Guri, Korea (the Republic of)

${ }^{8}$ Division of Pulmonology, National Cancer Center, Goyang, Korea (the Republic of) ${ }^{9}$ Department of Internal Medicine, National Medical Center, Seoul, Korea (the Republic of)

${ }^{10}$ Department of Internal Medicine, Dong-a University Hospital, Busan, Korea (the Republic of)

${ }^{11}$ Department of Internal Medicine, Seoul National University-Seoul Metropolitan Government Boramae Medical Center, Seoul National University College of Medicine, Dongjak-gu, Seoul, Korea (the Republic of)

${ }^{12}$ Department of Allergy, Pulmonary and Critical Care Medicine, CHA Bundang Medical Center, Sungnam, Korea (the Republic of)

${ }^{13}$ Division of Pulmonary and Critical Care Medicine, Department of Internal Medicine, Seoul National University Bundang Hospital, Sungnam, Korea (the Republic of)

${ }^{14}$ Division of Pulmonary and Critical Care Medicine, Department of Medicine, Samsung Medical Center, Sungkyunkwan University School of Medicine, Seoul, Korea (the Republic of)

${ }^{15}$ Division of Pulmonary and Critical Care Medicine, Department of Internal Medicine, Seoul National University Hospital, Seoul, Korea (the Republic of)

${ }^{16}$ Division of Pulmonary, Allergy and Critical Care Medicine, Department of Internal Medicine, College of Medicine, Seoul St Mary's Hospital, The Catholic University of Korea, Seoul, Korea (the Republic of)

${ }^{17}$ Department of Internal Medicine, Eunpyeong St. Mary's Hospital, College of Medicine, The Catholic University of Korea, Seoul, Korea (the Republic of)

${ }^{18}$ Department of Internal Medicine, Soonchunhyang University Cheonan Hospital, Cheonan, Korea (the Republic of)

${ }^{19}$ Department of Internal Medicine, Soonchunhyang University Bucheon Hospital, Bucheon, Korea (the Republic of)

${ }^{20}$ Division of Pulmonology, Department of Internal Medicine, Yonsei University Severance Hospital, Seoul, Korea (the Republic of)

${ }^{21}$ Division of Pulmonary Medicine, Department of Internal Medicine, Ulsan University Hospital, University of Ulsan College of Medicine, Ulsan, Korea (the Republic of)

${ }^{22}$ Department of Internal Medicine, Yonsei University Wonju College of Medicine, Wonju, Korea (the Republic of)

${ }^{23}$ Department of Internal Medicine, Jeju National University Hospital, Jeju, Korea (the Republic of) 
${ }^{24}$ Department of Internal Medicine, Hallym University Chuncheon Sacred Heart Hospital, Chuncheon, Korea (the Republic of)

${ }^{25}$ Division of Pulmonary and Critical Care Medicine, Department of Internal Medicine, Inje University Haeundae Paik Hospital, Busan, Korea (the Republic of)

${ }^{26}$ Department of Pulmonary and Critical Care Medicine, Asan Medical Center, University of Ulsan College of Medicine, Seoul, Korea (the Republic of)

Acknowledgements We would like to thank Professor James D Chalmers at the University of Dundee, Dundee, UK, for providing us the EMBARC platform and advice on the KMBARC registry. We would also like to thank Professor Jung Bok Lee at the University of Ulsan College of Medicine, Asan Medical Center, Seoul, Korea, for assistance in statistical analysis.

Collaborators Kim SH, Kwon YS, Kang HK, Kim KU, Kim YI, Kim YH, Kim J, Kim JW, Na MJ, Park SJ, Park SY, Park YB, Park HJ, Byun MK, Shin KC, Lee J, Lee JH, Lee JK, Lee HK, Lim SY, Jeon K, Jeong IB, Choi JS, Choi HS, Hong Y, Hwang YI.

Contributors $\mathrm{Y}-\mathrm{MO}$ is the principal investigator leading the KMBARC research and collaboration with all authors (HL, HC, YSS, SP, WJK, KHY, SJL, T-HK, BY, IJ, S-JU, DKK, J-HL, BSK, Y-JC, HYP, C-HL, CKR, SHL, J-ON, A-SJ, JYJ, SWR, J-HL, SHK, CK, YK, CYL, HKK, JSL and SWL). HL and HC drafted the first version of this manuscript. HL, HC, HYP, JSL, SWL and Y-MO conceived and designed the overall study. HL, $\mathrm{HC}$, HYP, JL, SWL and Y-MO are responsible for study oversight, management and coordination. All authors reviewed the manuscript for intellectual content and approved the final version of the report.

Funding This study was supported by a 2018 Grant from the Korean Academy of Tuberculosis and Respiratory Diseases.

Competing interests Y-M0 reports honorariums from GSK Korea, Novartis, Astra Zeneca Korea, MSD Korea, Boeheringer Ingelheim, Chong Kun Dang Pharm, MDimune and Daewoong. Y-MO also reports a research grant from Chong Kun Dang Pharm. All other authors declare no competing interests.

Patient consent for publication Not required.

Ethics approval This study received necessary approval from Institutional Review Boards of all participating institutions.

Provenance and peer review Not commissioned; externally peer reviewed.

Open access This is an open access article distributed in accordance with the Creative Commons Attribution Non Commercial (CC BY-NC 4.0) license, which permits others to distribute, remix, adapt, build upon this work non-commercially, and license their derivative works on different terms, provided the original work is properly cited, appropriate credit is given, any changes made indicated, and the use is non-commercial. See: http://creativecommons.org/licenses/by-nc/4.0/.

\section{ORCID iDs}

Hayoung Choi http://orcid.org/0000-0003-4812-0653

Yeon-Mok Oh http://orcid.org/0000-0003-0116-4683

\section{REFERENCES}

1 Polverino E, Goeminne PC, McDonnell MJ, et al. European respiratory Society guidelines for the management of adult bronchiectasis. Eur Respir J 2017;;50:1700629.

2 Martínez-García MA, Perpiñá-Tordera M, Román-Sánchez P, et al. Quality-Of-Life determinants in patients with clinically stable bronchiectasis. Chest 2005;128:739-45.

3 Goeminne PC, Hernandez F, Diel R, et al. The economic burden of bronchiectasis - known and unknown: a systematic review. BMC Pulm Med 2019;19:54

4 Chalmers JD, Aliberti S, Polverino E, et al. The EMBARC European bronchiectasis registry: protocol for an international observational study. ERJ Open Res 2016;2:00081-2015-2015.

5 Aksamit TR, O'Donnell AE, Barker A, et al. Adult patients with bronchiectasis: a first look at the US bronchiectasis research registry. Chest 2017;151:982-92.

6 Choi H, Yang B, Nam H, et al. Population-Based prevalence of bronchiectasis and associated comorbidities in South Korea. Eur Respir J 2019;54:1900194.

7 Kroenke K, Spitzer RL, Williams JB. The PHQ-9: validity of a brief depression severity measure. J Gen Intern Med 2001;16:606-13.

8 Krupp LBet al. The fatigue severity scale. Arch Neurol 1989;46:1121-3.

9 Hill AT, Haworth CS, Aliberti S, et al. Pulmonary exacerbation in adults with bronchiectasis: a consensus definition for clinical research. Eur Respir J 2017;49:1700051.
10 Pasteur MC, Bilton D, Hill AT, et al. British thoracic Society guideline for non-CFbronchiectasis. Thorax 2010;65:i1-58.

11 Murray MP, Pentland JL, Turnbull K, et al. Sputum colour: a useful clinical tool in non-cystic fibrosis bronchiectasis. Eur Respir J 2009;34:361-4.

12 Miller MR, Hankinson J, Brusasco V, et al. Standardisation of spirometry. Eur Respir J 2005;26:319-38.

13 Choi JK, Paek D, Lee J, et al. Normal predictive values of spirometry in Korean population 2005:58:230-42.

14 Chalmers JD, Goeminne P, Aliberti S, et al. The bronchiectasis severity index. An international derivation and validation study. Am J Respir Crit Care Med 2014;189:576-85.

15 Cockerill F, Wikler M, Bush K. Performance standards for antimicrobial susceptibility testing; 21st informational supplement. Wayne, PA: Clinical and Laboratory Standards Institute, 2011.

16 King PT, Holdsworth SR, Freezer NJ, et al. Microbiologic follow-up study in adult bronchiectasis. Respir Med 2007;101:1633-8.

17 Martínez-García MA, Soler-Cataluña J-J, Perpiñá-Tordera M, et al. Factors associated with lung function decline in adult patients with stable non-cystic fibrosis bronchiectasis. Chest 2007;132:1565-72.

18 Reiff DB, Wells AU, Carr DH, et al. Ct findings in bronchiectasis: limited value in distinguishing between idiopathic and specific types. AJR Am J Roentgenol 1995;165:261-7.

19 Haworth CS, Foweraker JE, Wilkinson P, et al. Inhaled Colistin in Patients with Bronchiectasis and Chronic Pseudomonas aeruginosa Infection. Am J Respir Crit Care Med 2014;189:975-82.

20 Roberts HJ, Hubbard R. Trends in bronchiectasis mortality in England and Wales. Respir Med 2010;104:981-5.

21 Ringshausen FC, de Roux A, Pletz MW, et al. BronchiectasisAssociated hospitalizations in Germany, 2005-2011: a population-based study of disease burden and trends. PLoS One 2013;8:e71109.

22 Diel R, Chalmers JD, Rabe KF, et al. Economic burden of bronchiectasis in Germany. Eur Respir J 2019;53:1802033.

23 Ringshausen FC, de Roux A, Diel R, et al. Bronchiectasis in Germany: a population-based estimation of disease prevalence. Eur Respir J 2015;46:1805-7.

24 Seitz AE, Olivier KN, Adjemian J, et al. Trends in bronchiectasis among medicare beneficiaries in the United States, 2000 to 2007. Chest 2012;142:432-9.

25 Qi Q, Wang W, Li T, et al. Aetiology and clinical characteristics of patients with bronchiectasis in a Chinese Han population: a prospective study. Respirology 2015;20:917-24.

26 Mohan M, D'souza G, Rajagopalan S, et al. Phenotype characterization of non cystic fibrosis bronchiectasis in India: baseline data from an Indian bronchiectasis registry. Am J Respir Crit Care Med 2017;195:A4726.

27 Guan W-jie, Gao Y-hua, Xu G, et al. Aetiology of bronchiectasis in Guangzhou, southern China. Respirology 2015;20:739-48.

28 Chan-Yeung M, Lai CKW, Chan KS, et al. The burden of lung disease in Hong Kong: a report from the Hong Kong thoracic Society. Respirology 2008;13:S133-65.

29 Chandrasekaran R, Mac Aogáin M, Chalmers JD, et al. Geographic variation in the aetiology, epidemiology and microbiology of bronchiectasis. BMC Pulm Med 2018;18:83.

30 Dhar R, Singh S, Talwar D, et al. Bronchiectasis in India: results from the European multicentre bronchiectasis audit and research collaboration (EMBARC) and respiratory research network of India registry. Lancet Glob Health 2019;7:e1269-79.

31 Agusti A, Calverley PMA, Celli B, et al. Characterisation of COPD heterogeneity in the eclipse cohort. Respir Res 2010;11:122.

32 Couper D, LaVange LM, Han M, et al. Design of the Subpopulations and Intermediate Outcomes in COPD Study (SPIROMICS): Table 1. Thorax 2014;69:492-5

33 Lee JY, Chon GR, Rhee CK, et al. Characteristics of patients with chronic obstructive pulmonary disease at the first visit to a pulmonary medical center in Korea: the Korea COPD subgroup study Team cohort. J Korean Med Sci 2016;31:553-60.

34 Park TS, Lee JS, Seo JB, et al. Study design and outcomes of Korean obstructive lung disease (KOLD) cohort study. Tuberc Respir Dis 2014;76:169-74

35 Regan EA, Hokanson JE, Murphy JR, et al. Genetic epidemiology of COPD (COPDGene) study design. COPD 2011;7:32-43.

36 Vestbo J, Anderson W, Coxson HO, et al. Evaluation of COPD longitudinally to identify predictive surrogate end-points (eclipse). Eur Respir J 2008;31:869-73.

37 Chalmers JD, Crichton M, Goeminne PC, et al. The European multicentre bronchiectasis audit and research collaboration (EMBARC): experiences from a successful ERS clinical research collaboration. Breathe 2017;13:180-92. 
38 McDonnell MJ, Aliberti S, Goeminne PC, et al. Comorbidities and the risk of mortality in patients with bronchiectasis: an international multicentre cohort study. Lancet Respir Med 2016;4:969-79.
39 Jeong $\mathrm{HJ}$, Lee $\mathrm{H}$, Carriere KC, et al. Effects of long-term bronchodilators in bronchiectasis patients with airflow limitation based on bronchodilator response at baseline. Int J Chron Obstruct Pulmon Dis 2016;11:2757-64. 